\title{
The Association between Neuroticism and Re-Attempted Suicide
}

Probert-Lindström S* and Ambrus L

Department of Clinical Sciences, Lund, Psychiatry, Lund

University, Clinical Psychiatric Research Center, Region Skåne, Sweden

*Corresponding author: Sara Probert-Lindström, Psykiatriforskning Skåne, Baravägen 1, S-221 85 Lund, Sweden

Received: November 19, 2021; Accepted: December 10, 2021; Published: December 17, 2021

\begin{abstract}
Background: Strong evidence suggests that suicide attempters who repeat suicide (re-attempters) may differ from those with a single attempt (single attempters) in various clinical and sociodemographic factors. Furthermore, there is some evidence that re-attempters may be characterized by higher levels of neuroticism, a well-known risk factor for completed suicide compared to single attempters.
\end{abstract}

Purpose: The aim of the present study was to investigate the possible association between neuroticism and re-attempted suicide.

Methods and Materials: 230 recent drug-free suicide attempters were involved into the study. Clinical diagnoses were assessed by the Diagnostic and Statistical Manual of Mental Disorders, Third Edition-Revised (DSM-III-R). Personality traits were measured by the Karolinska Scale of Personality (KSP).

Results and Conclusions: There was a significant association between re-attempted suicide, female gender, personality disorder and higher levels of impulsivity. We found that re-attempters had significantly higher levels of neuroticism compared to single attempters adjusted of gender, personality disorder diagnosis, age and impulsivity. Our result gives further evidence for the association between re-attempted suicide and neuroticism.

Keywords: Repeated suicide attempt; Neuroticism; Impulsivity; Karolinska Scale of Personality

\section{Introduction}

Suicide is a major health issue throughout the world and one of the leading causes of death among young people [1]. Every year more than 800,000 people die of suicide [2] including 1,200 people in Sweden [3]. Suicide is a traumatic and tragic loss for the close family and friends of the victim. It also represents a significant loss to society as a whole. In 2014 alone, suicides in Sweden resulted in the loss of 38,000 lived years at an estimated direct and indirect cost to the country of 10-40 billion SEK (corresponding to about 1-4 billion USD or Euro) [4].

Health care professionals have tended to view suicidal behavior as a symptom or effect of underlying psychiatric conditions and this focus may actually hamper prevention efforts. However, the majority of individuals with a psychiatric diagnosis does not present with suicidal ideation nor do they ever complete a suicide attempt. It is also true that suicidal behaviors occur in individuals without obvious signs of (or a diagnosis of) any psychiatric illness. While more research is needed, there is a growing recognition that suicide is not necessarily the downstream effects of another psychiatric disorder but its own disorder, which may share risk factors with other psychiatric disorders $[5,6]$. Approximately half of those who commit suicide every year in Sweden sought help from their primary or secondary (psychiatric) health care provider just prior to the suicide, indicating that further improvements are needed in screening and prevention efforts [7].
The pathophysiology of suicide is a complex process in which genetic, neurobiological, environmental and psychological factors interacts [8]. During the last decades, several risk factors for completed suicide have been identified including one of the most promising diagnosis-independent risk factors is a previous suicide attempt $[9,10]$. However, as not all suicide attempters die by suicide, identifying risk factors of subsequent suicide and understanding underlying mechanisms among suicide attempters is vital and can aid clinicians in the challenging task of suicide risk assessment. Robust evidence suggests that suicide attempters who re-attempt suicide (re-attempters) had a significantly higher risk of subsequent suicide compared to those who do not repeat suicide attempt (single attempters) [11-16]. Moreover, the increased risk of suicide among re-attempters may be independent of the intentionality of the previous suicide attempt [17] and appears to remain an important risk factor for suicide for more than 30 years [18].

Several studies comparing re-attempters and single attempters have reported that re-attempters may have specific clinical and sociodemographic characteristics [19-23]. Particularly, re-attempters are younger, are more often single [23], have a family history of suicide $[19,21,23]$, have experienced childhood sexual or emotional abuse [20,21], and have a poorer social and interpersonal function [21,23]. Furthermore, re-attempters appear to have a higher prevalence of clinical disorders [23] including Major Depressive Disorder (MDD), substance abuse disorder or Personality Disorder (PD) [20-22] and have more severe clinical symptoms of hopelessness 
compared to single attempters $[11,19]$. Previous research have shown that certain personality traits like neuroticism appear to be associated with increased suicide risk of completed suicide [24]. There is also evidence for differences in personality traits like neuroticism between re-attempters and single attempters [25]. Specifically, Pennel and his co-workers found that re-attempters had higher levels of neuroticism compared to single attempters [25]. Neuroticism is a relatively stable personality trait characterized by increased reactivity to negative stimuli and are associated with experiences such as anxiety, worry, frustration, anger, depressed mood, and impulsiveness [26]. Evidence suggests even that higher levels of neuroticism contribute to the risk of mental disorders and suicide [27]. In sum, the finding of Pennel and colleagues indicates that higher levels of neuroticism may contribute to the higher risk of completed suicide among reattempters. However, further studies investigating the association between re-attempted suicide and neuroticism are needed before conclusions can be drawn.

The aim of this study was to investigate the putative association between re-attempted suicide and neuroticism with the hypothesis that re-attempters have higher levels of neuroticism, compared to single attempters.

\section{Materials and Methods}

Participants were recruited between 1987 and 2001, shortly after a suicide attempt at the University Hospital in Lund. Approximately 190 suicide attempters were admitted to the MIEU every year during the study period. The number of suicide attempters who did not seek emergency care is impossible to estimate. Individuals who were admitted on weekends, evenings or holidays were not assessed for inclusion, representing approximately two thirds of all admitted. Of the individuals assessed during weekdays, approximately half were referred to the psychiatric research ward. Of those admitted, about 91\% agreed to participate in the study. Patients in need of immediate psychiatric treatment were not included and some patients were missed due to high work load at the ward.

\section{Inclusion criteria}

- $\quad$ Age over 18 years.

- Admission to the Medical Emergency and Intensive care Unit (MEIU) after an attempted suicide. As suicide attempts were considered as 'situations in which a person has performed an actually or seemingly life-threatening behaviour with the intent of jeopardizing his/her life, or to give the appearance of such an intent but which has not resulted in death' [28].

- $\quad$ From the MEIU referred to the psychiatric research ward and admitted for at least one week.

\section{Exclusion criteria}

- Discharge before information and consent about the study.

- Urgent need for psychiatric treatment (i.e. psychotic symptoms or severe depressive symptoms and therefore washout not possible).

For this study, of the 304 patients remaining, 45 did not fill the Karolinska Scale of Personality (KSP) (KSP; please see below). Further, 8 were excluded due to lack of data regarding previous suicide attempts. Another 3 lacked data of psychiatric diagnosis and additional 18 individuals were lacking data on personality disorder diagnosis, leaving 230 individuals for inclusion in this study.

All patients gave their informed consent to participate in the study (LU14-1988). Psychiatric diagnoses were set by consensus by two trained psychiatrists who independently diagnosed the patients according to the Diagnostic and Statistical Manual of Mental Disorders, Third Edition-Revised (DSM-III-R). All patients filled in the KSP. The patients did not receive any psychopharmacologic treatment after the suicide attempt. A re-attempter refers to individuals who had a history of previous attempts at suicide before the index suicide attempt.

Patients were divided into violent or non-violent suicide attempters according to the previously described classification [29]. Violent method refers to the method of attempted suicide; either a method other than drug overdose or single wrist-cut, or a combination of different methods. Data regarding clinical, demographic variables and personality traits are presented in Table 1 . The previously reported characteristics of re-attempters such as marital status, family history of suicide, childhood abuse and social and interpersonal functioning was not analyzed in this study due to lack of data on these variables.

\section{The Karolinska scale of personality}

To assess personality traits, we used the Karolinska Scale of Personality (KSP) [30]. This instrument is a widely used measure of temperament/personality. The questionnaire is a self-report inventory developed by Schalling et al. with the purpose of measuring vulnerability for different forms of psychopathology. KSP comprises 135-items with four-point Likert scale from 1 "Does not apply at all" to 4 Applies completely. The KSP is further described in Engström et al. [31]. The items are grouped into 15 scales such as Somatic anxiety, Muscular tension, Psychic anxiety, Psychasthenia, Inhibition of aggression, Suspicion, Guilt, Socialization, Social desirability, Indirect aggression, Verbal aggression, Irritability, Impulsiveness, Monotone avoidance and Detachment. These factors were factor analyzed by among others Schalling et al. [32] resulting five factors including Hostility (Suspicion and Guilt), Psychopathy vs. conformity (Socialization and Social desirability), Aggressivity (Indirect aggression, Verbal aggression, Irritability), Impulsivity (Impulsiveness, Monotone avoidance, Detachment) and Neuroticism (Somatic anxiety, Muscular tension, Psychic anxiety, Psychasthenia, Inhibition of aggression). To investigate neuroticism the Neuroticism factor (named in the present study as Neuroticism subscale) was used. This subscale are presented in Table 2.

\section{Statistics}

Statistical analyses were made using IBM SPSS Statistics 21.0 [33]. Data regarding age was not normally distributed, even after logtransformation. In order to be able to use age as a covariate, data was dichotomized using the mean of age [38] as the cut-off. In order to identify possible covariates, we investigated the association between the history of a previous suicide attempt and various clinical and demographic variables such as age, gender, $\mathrm{PD}$, an MDD diagnosis, anxiety disorders, psychotic disorders, dysthymia, substance abuse disorders and the personality traits such as Hostility, Aggressivity, Impulsivity and Psychopathy with Fisher's exact test (between dichotomous data), Pearson's correlation (between linear data) and 
Table 1: The Neuroticism subscale of the KSP.

\begin{tabular}{|l|l|l|}
\hline \multicolumn{1}{|c|}{ KSP scales [32] } & \multicolumn{1}{c|}{ Description } \\
\hline Somatic anxiety & Autonomic symptoms, concentration difficulties, vague distress, and panic \\
\hline Muscular tension & Tenseness in muscles, trembling, not relaxed \\
\hline Psychic anxiety & Worry, insecurity, anticipatory and social anxiety \\
\hline Psychasthenia & Being easily fatigued, feeling uneasy when urged to speed up when facing new tasks \\
\hline Inhibition of aggression & Non-assertive, sad rather than angry when scolded, and cannot speak up \\
\hline
\end{tabular}

Table 2: Demographic and clinical variables of the sample.

\begin{tabular}{|c|c|c|c|c|}
\hline & All patients & \multirow{2}{*}{$\%$} & Single attempters & \multirow{2}{*}{$\begin{array}{c}\text { Re-attempters } \\
\text { N=95 }\end{array}$} \\
\hline & $\mathrm{N}=\mathbf{2 3 0}$ & & $\mathrm{N}=135$ & \\
\hline Age (mean \pm SD) & $37.9 \pm 13.5$ & - & $37.7 \pm 13.7$ & $38.2 \pm 12.8$ \\
\hline Gender Males/Females & $103 / 127$ & $45 / 55$ & $64 / 71$ & $63 / 32$ \\
\hline Violent method Yes/No & $51 / 179$ & $22 / 78$ & $34 / 101$ & $17 / 78$ \\
\hline \multicolumn{5}{|l|}{ Main axis I disorder } \\
\hline MDD Yes/No & $85 / 145$ & $37 / 63$ & $48 / 87$ & $37 / 58$ \\
\hline Dysthymia Yes/No & $31 / 199$ & $13 / 87$ & $14 / 121$ & $17 / 78$ \\
\hline Substance abuse disorder Yes/No & $39 / 191$ & $17 / 83$ & $19 / 116$ & $20 / 75$ \\
\hline Anxiety disorders & $10 / 220$ & $4 / 96$ & $6 / 129$ & $4 / 91$ \\
\hline Psychotic disorder & $11 / 219$ & $5 / 95$ & $7 / 128$ & $4 / 91$ \\
\hline PD Yes/No & $95 / 135$ & $41 / 59$ & $79 / 56$ & $70 / 25$ \\
\hline
\end{tabular}

Abbreviations: SD: Standard Deviation; MDD: Major Depressive Episode; PD: Personality Disorder.

Student's test (between linear and dichotomous data). Secondly, we studied the association between Neuroticism scale and the clinical and demographic variables such as age, gender, dysthymia, $\mathrm{PD}$, an MDD diagnosis, substance abuse disorders with the aim to find possible covariates. The linear regression analysis was used to study the possible association between re-attempted suicide (as an independent variable) and Neuroticism subscale (as the dependent variable). Variables found to associate with re-attempted suicide or Neuroticism subscale were also included into the regression analysis as independent variables. Two-tailed level of significance was used. Alpha-level of significance was set at $\mathrm{p}<0.05$.

\section{Ethical approval statement}

The study was approved by the Lund University Medical Ethics Committee. All participants gave their informed consent to participate in the study (LU14-1988).

\section{Results}

Comparison of clinical and demographic variables between re-attempters and single attempters

The clinical, demographic variables and the Neuroticism subscale regarding the history of attempted suicide are presented in Table 2. The mean (mean $\pm \mathrm{SD}$ ) of remaining personality traits among reattempers were the following: Impulsivity: $163.9 \pm 21.3$, Aggressivity: $154.3 \pm 25.2$, Psychopathy vs. conformity: $77.5 \pm 19.5$ and Hostility $117.5 \pm 19.1$. The mean (mean \pm SD) of remaining personality traits among single attempters were the following: Impulsivity: $156.0 \pm$ 23.2, Aggressivity: $154.5 \pm 23.4$, Psychopathy vs. nonconformity: 81.3 \pm 18.3 and Hostility: $112.5 \pm 18.0$.

Re-attempters were significantly more often females $(p=0.005)$ and had more often PD $(p=0.040)$ compared to single attempters. No significant differences in the use of a violent method $(p=0.190)$, age $(p=0.608)$, clinical diagnoses such as $\operatorname{MDD}(p=0.600)$, psychotic disorders $(p=0.465)$, anxiety disorders $(p=0.775)$, dysthymia $(p=0.100)$ or substance abuse disorders $(p=0.208)$ were observed between reattempters and single attempters.

Re-attempters had significantly higher scores on Impulsivity subscale $(p=0.010)$ compared to single attempters. Re-attempters were comparable in the scores of Psychopathy vs. noncormity $(p=0.132)$, Hostility $(p=0.173)$ and Aggressivity subscales $(p=0.951)$.

Correlations between the Neuroticism subscale, clinical and demographic variables

The scores of Neuroticism subscale were significantly correlated with age $(p=0.002)$ and PD $(p=0.001)$ but not with gender $(p=0.206)$, $\operatorname{MDD}(p=0.658)$, psychotic disorders $(p=0.999)$, anxiety disorders $(p=0.304)$, dysthymia $(p=0.119)$, substance abuse disorders $(p=0.208)$ or the use of violent method ( $p=0.774)$.

\section{Neuroticism and re-attempted suicide}

The linear regression analysis showed that re-attempters had significantly higher scores on the Neuroticism subscale $(B=0.151$, $p=0.024)$ adjusted of age, gender, Impulsivity and PD.

\section{Discussion}

In line with our hypothesis, we found that re-attempters are characterized by higher levels of neuroticism compared to single attempters adjusted for age, PD, gender and impulsivity. This result is similar to Pennel and co-workers reporting higher levels of neuroticism among re-attempters compared to single attempters 
[25]. Interestingly, in a previous study, a 5-year follow-up of parts of the population in the current study [34] showed that KSP neuroticism scores decreased compared to baseline scores among suicide attempters with a single known attempt, but remained more stable over time among re-attempters (i.e. individuals who re-attempted during follow-up). Taken together, these results may indicate that higher levels of neuroticism may represent a vulnerability to repeat suicide attempts. Interestingly, as a secondary, re-attempters was also found to have higher levels on impulsivity compared to single attempters. It has been previously shown that impulsivity is associated with suicidal behaviour [35], though to the best of our knowledge, the connection between impulsivity and repeated attempts has not previously been documented in the literature. However, before drawing any conclusion, new studies investigating the association between impulsivity and re-attempted suicide are needed.

Our study may have some important implications for both researchers and clinicians. Firstly, our finding raises the question of whether the assessment of neuroticism among suicide attempters could be used in order to predict subsequent suicide attempts or suicide among suicide attempters. Therefore, it would be of interest to perform prospective studies to test this research question. In addition, our finding may raise the issue of whether psychotherapy with a focus on improving neuroticism could reduce the risk of suicide in suicide attempters. Several benefits of focusing treatment efforts on underlying psychological vulnerability rather than targeting psychiatric diagnosis have been proposed, among them the fact that psychiatric comorbidity is high and many symptoms are shared between conditions [36]. Treatment that specifically target neuroticism like for instance transdiagnostic treatment protocol Unified protocol [37], have proven efficient - contrary to the common understanding of the stability of personality traits [36].

Not only psychotherapy but some psychopharmacological treatments have been found to decrease neuroticism. Specifically, previous studies reported that paroxetine and escitalopram belonging to serotonin reuptake inhibitors (SSRI), a group of antidepressant led to a decreasing in neuroticism [38-40]. The pathophysiology of neuroticism is not clear; however, the dysfunction of the serotonergic system have been repeatedly linked to neuroticism [41]. Based on this fact, the possible mechanism underlying SSRI's effect on neuroticism is that these molecules may diminish the serotonergic dysfunction underlying neuroticism [41].

Further, the present study found that re-attempters were diagnosed with PD significantly more often than single attempters. This finding is in line with previous results and strengthens the evidence for the association between PD and reattempted suicide [22,25].

\section{Limitations and Strengths}

There are several limitations to this study. The main limitation is the relatively small sample size and the absence of controls. Furthermore, being a cross-sectional study, this study did not have data on whether or not the single suicide attempters, later made additional suicide attempts. Hence, an unknown portion of the single suicide attempters may later have become re-attempters. It is therefore likely that the group of re-attempters is actually larger and the group of single attempters accordingly smaller. The results should be interpreted in light of this. Another limitation is the use of selfreport measures, including data regarding previous suicide attempts that rely solely on patient reports. This method has an inherent risk of bias as many individuals are influenced by social desirability i.e. they are more likely to report experiences considered to be socially acceptable [42]. Further, generalizability to the wider population of individuals who attempt suicide is limited as this sample consists of individuals who were admitted to a MEIU as a consequence of attempted suicide, indicating a certain medical severity of the attempt. Another limitation is that we did not take a count other factors with importance for both suicidal risk and personality traits like childhood traumatic experience. Childhood trauma has been linked directly to the increased risk of completed suicide, as well to vulnerability factors for suicide like depressive or anxiety symptom, dysfunctional sensory perception and neuroticism [43-45]. Further, another limitation is the difficulty of diagnostic assessment in an inpatient setting after a suicide attempt, though the assessors were all experienced psychiatrists and two psychiatrists assessed all patients independently of each other. Finally, it is important to note the likely highly complex associations between aspects of an individual's traits and behavior such as suicide attempts. This study can only aspire to shed lights on observed associations to be further investigated and more thoroughly understood.

It is well-known, that psychopharmacological treatment like benzodiazepines, anticonvulsants, sedatives and antipsychotics have side effect including cognitive impairment [46-49]. There is also evidence that the acute administration of hypnotics like zolpidem/ triazolam affects the cognitive performance 12 hours after acute administration [49]. In addition, according to some evidence, antidepressant may lead to changes in personality traits including neuroticism and extraversion [50-52]. Therefore, the fact that individuals in the present study are free from psychopharmacologic is a strength.

\section{Conclusion and Main Implications}

The results of the present study give further evidence for the association between neuroticism and reattempted suicide. An association, previously undocumented, was also found between impulsivity and repeated suicide attempts. Both neuroticism and impulsivity have been shown to be malleable by treatment efforts, both psychological and pharmacological. It remains unclear whether interventions aimed at these aspects of functioning could prove effective in preventing repeated suicide attempts. It is possible that screening for neuroticism and impulsivity could be helpful suicide risk assessment among suicide attempters. Further research is warranted to test if such clinical routines could aid clinicians in the challenging task of suicide risk assessment.

\section{Declaration}

Acknowledgment: The authors are grateful to Professor Lil Träskman-Bendz, the principal investigator for the study. This work was supported by the South Region Board, Region Skåne.

Data availability statement: Data available on request from the authors. 
Author contribution: LA: Designed the study and made the analysis. LA and SPL: Interpreted the data. LA and SPL: Drafted the paper. LA and SPL: Revised it. LA and SPL: Approved of the final version of the paper and agree to be accountable for all aspects of the work.

\section{References}

1. World Health Organization. Preventing suicide. 2014.

2. WHO. Suicide rates (per 100000 population).

3. Socialstyrelsen. Suicide rates (per 100000 population). 2018.

4. MSB. Samhällsekonomiska konsekvenser av fullbordade suicide. 2015

5. Ellis TE, Goldston DB. Working With Suicidal Clients: "Not" Business as Usual. Cognitive and Behavioral Practice. 2012; 19: 205-208.

6. Ahrens B, Linden M, Zäske H, Berzewski H. Suicidal behavior-symptom or disorder? Comprehensive Psychiatry. 2000; 41: 116-121.

7. Martin-Carrasco M, Evans-Lacko S, Dom G, Christodoulou NG, Samochowiec $\mathrm{J}$, González-Fraile E, et al. EPA guidance on mental health and economic crises in Europe. European Archives of Psychiatry and Clinical Neuroscience. 2016; 266: 89-124.

8. Domenico De B, Michele F, Alessandro V, Marilde C, Giampaolo P, Marco Di N, et al. Eradicating Suicide at Its Roots: Preclinical Bases and Clinical Evidence of the Efficacy of Ketamine in the Treatment of Suicidal Behaviors. International journal of molecular sciences. 2018; 19: 2888.

9. Bostwick JM, Pabbati C, Geske JR, McKean AJ. Suicide Attempt as a Risk Factor for Completed Suicide: Even More Lethal Than We Knew. American Journal of Psychiatry. 2016; 173: 1094-1100.

10. Suominen K, Isometsä E, Suokas J, Haukka J, Achte K, Lönnqvist J. Completed Suicide after a Suicide Attempt: A 37-Year Follow-Up Study. American Journal of Psychiatry. 2004; 161: 562-563.

11. Menon V, Kattimani S, Sarkar S, Mathan K. How do repeat suicide attempters differ from first timers? An exploratory record based analysis. Journal of Neurosciences in Rural Practice. 2016; 7: 91-96.

12. Cedereke M, Öjehagen A. Prediction of repeated parasuicide after 1-12 months. European Psychiatry. 2005; 20: 101-109.

13. De Leo D, Padoani W, Lonnqvist J, Kerkhof AJFM, Bille-Brahe U, Michel K, et al. Repetition of suicidal behaviour in elderly Europeans: a prospective longitudinal study. Journal of affective disorders. 2002; 72: 291-295.

14. Chandrasekaran R, Gnanaselane J. Predictors of Repeat Suicidal Attempts after First-ever Attempt: a Two-year Follow-up Study. Hong Kong Journal of Psychiatry. 2008; 18: 131-135.

15. Kotila L, Lönnqvist J. Adolescents who make suicide attempts repeatedly. Acta psychiatrica Scandinavica. 1987; 76: 386-393.

16. Holmstrand C, Niméus A, Träskman-Bendz L. Risk factors of future suicide in suicide attempters--a comparison between suicides and matched survivors. Nordic Journal of Psychiatry. 2006; 60: 162-167.

17. Andover MS, Gibb BE. Non-suicidal self-injury, attempted suicide, and suicidal intent among psychiatric inpatients. Psychiatry Research. 2010; 178 101-105.

18. Probert-Lindström S, Berge J, Westrin Å, Öjehagen A, Skogman Pavulans K Long-term risk factors for suicide in suicide attempters examined at a medical emergency in patient unit: results from a 32-year follow-up study. BMJ Open. 2020; 10: e038794.

19. Liu Y, Zhang J, Sun L. Who are likely to attempt suicide again? A comparative study between the first and multiple timers. Comprehensive Psychiatry. 2017; 78: 54-60.

20. Kochanski-Ruscio KM, Carreno-Ponce JT, DeYoung K, Grammer G, Ghahramanlou-Holloway M. Diagnostic and psychosocial differences in psychiatrically hospitalized military service members with single versus multiple suicide attempts. Comprehensive Psychiatry. 2014; 55: 450-456.
21. Forman EM, Berk MS, Henriques GR, Brown GK, Beck AT. History of multiple suicide attempts as a behavioral marker of severe psychopathology. American Journal of Psychiatry. 2004; 161: 437-443.

22. Blasco-Fontecilla H, Rodrigo-Yanguas M, Giner L, Lobato-Rodriguez MJ, de Leon J. Patterns of Comorbidity of Suicide Attempters: An Update. Current Psychiatry Report. 2016; 18: 93.

23. Choi KH, Wang S-M, Yeon B, Suh S-Y, Oh $\mathrm{Y}$, Lee $\mathrm{H}-\mathrm{K}$, et al. Risk and protective factors predicting multiple suicide attempts. Psychiatry research. 2013; 210: 957-961.

24. Pompili M, Rihmer Z, Akiskal HS, Innamorati M, lliceto $P$, Akiskal KK, et al. Temperament and Personality Dimensions in Suicidal and Nonsuicidal Psychiatric Inpatients. Psychopathology. 2008; 41: 313-321.

25. Pennel L, Quesada J-L, Dematteis M. Neuroticism and anxious attachment as potential vulnerability factors of repeat suicide attempts. Psychiatry Research. 2018; 264: 46-53.

26. Costa PT, McCrae RR. Four ways five factors are basic. Personality and Individual Differences. 1992; 13: 653-665.

27. Brezo J, Paris J, Turecki G. Personality traits as correlates of suicidal ideation, suicide attempts, and suicide completions: a systematic review. Acta Psychiatrica Scandinavica. 2006; 113: 180-206.

28. Beck AT, Davis J, Frederick CJ, Perlin S, Pokorny A, Schulman R, et al. Classification and nomenclature; in Resnik HPL, Hathorne B (eds): Suicide Prevention in the Seventies. US Government Printing Office, Washington, DC. 1972.

29. Rydin E, Asberg M, Edman G, Schalling D. Violent and nonviolent suicide attempts--a controlled Rorschach study. Acta Psychiatrica Scandinavia. 1990; 82: 30-39.

30. Schalling D, Åsberg M, Edman G, Oreland L. Markers for vulnerability to psychopathology: Temperament traits associated with platelet MAO activity. Acta Psychiatrica Scandinavica. 1987; 76: 172-182.

31. Engström G, Alling C, Gustavsson P, Oreland L, Träskman Bendz L, Engstrom $G$, et al. Clinical characteristics and biological parameters in temperamental clusters of suicide attempters. Journal of Affective Disorders. 1997; 44: 45.

32. Ortet G, Ibáñez MI, Llerena A, Torrubia R. The underlying traits of the Karolinska Scales of Personality (KSP). European Journal of Psychological Assessment. 2002; 18: 139-148.

33. Norusis M. SPSS for windows. SPSS Inc, Chicago. 1995

34. Öjehagen A, Johnsson E, Träskman-Bendz L. The long-term stability of temperament traits measured after a suicide attempt. A 5-year follow-up of ratings of Karolinska scales of personality (KSP)/the long-term stability of temperament traits measured after a suicide attempt. A 5-year followup of ratings of Karolinska scales of personality (KSP). Nordic Journal of Psychiatry. 2003: 125.

35. Horesh N, Gothelf D, Ofek H, Weizman T, Apter A. Impulsivity as a correlate of suicidal behavior in adolescent psychiatric inpatients. Crisis. 1999; 20: 8-14.

36. Sauer-Zavala S, Wilner JG, Barlow DH. Addressing neuroticism in psychological treatment. Personality Disorders: Theory, Research, and Treatment. 2017; 8: 191-198.

37. Barlow DH. Unified protocol for transdiagnostic treatment of emotional disorders. 2 ed. ed: Oxford University Press. 2017.

38. Andrews W, Parker G, Barrett E. The SSRI antidepressants: exploring their "other" possible properties. Journal of Affective Disorders. 1998; 49: 141-144.

39. Tang TZ, DeRubeis RJ, Hollon SD, Amsterdam J, Shelton R, Schalet B. Personality change during depression treatment: a placebo-controlled trial. Archives of General Psychiatry. 2009; 66: 1322-1330.

40. Du L, Bakish D, Ravindran AV, Hrdina PD. Does fluoxetine influence majo depression by modifying five-factor personality traits? Journal of Affective Disorders. 2002; 71: 235-241.

41. Foster JA, MacQueen G. Neurobiological factors linking personality traits 
and major depression. Canadian journal of psychiatry Revue canadienne de psychiatrie. 2008; 53: 6-13.

42. Sjöberg L. Correction for faking in self-report personality tests. Scandinavian journal of psychology. 2015; 56: 582-591.

43. Zatti C, Rosa V, Barros A, Valdivia L, Calegaro VC, Freitas LH, et al. Childhood trauma and suicide attempt: A meta-analysis of longitudinal studies from the last decade. Psychiatry Research. 2017; 256: 353-358.

44. Brodsky BS. Early Childhood Environment and Genetic Interactions: the Diathesis for Suicidal Behavior. Current Psychiatry Report. 2016; 18: 86

45. Serafini G, Gonda X, Pompili M, Rihmer Z, Amore M, Engel-Yeger B. The relationship between sensory processing patterns, alexithymia, traumatic childhood experiences, and quality of life among patients with unipolar and bipolar disorders. Child abuse \& neglect. 2016; 62: 39-50.

46. Stewart SA. The effects of benzodiazepines on cognition. The Journal of clinical psychiatry. 2005; 66: 9-13.

47. Goldberg JF, Burdick KE. Cognitive side effects of anticonvulsants. The Journal of clinical psychiatry. 2001; 62: 27-33.
48. Cullen B, Ward J, Graham NA, Deary IJ, Pell JP, Smith DJ, et al. Prevalence and correlates of cognitive impairment in euthymic adults with bipolar disorder: A systematic review. Journal of Affective Disorders. 2016; 205: 165-181.

49. Matsunaga $Y$, Tagaya $H$, Fukase $Y$, Hakamata $Y$, Murayama $N$, Kumagai $Y$, et al. Effects of zolpidem/triazolam on cognitive performance 12 hours after acute administration. Sleep medicine. 2018; 52: 213-218.

50. Võhma Ü, Raag M, Tõru I, Aluoja A, Maron E. Association between personality traits and Escitalopram treatment efficacy in panic disorder. Nordic Journal of Psychiatry. 2017; 71: 433-440.

51. Ekselius L, von Knorring L. Changes in personality traits during treatment with sertraline or citalopram. The British Journal of Psychiatry. 1999; 174 444-448.

52. Bagby RM, Levitan RD, Kennedy SH, Levitt AJ, Joffe RT. Selective alteration of personality in response to noradrenergic and serotonergic antidepressant medication in depressed sample: evidence of non-specificity. Psychiatry Research. 1999: 211-216. 